\title{
A Numerical Method for Electrical Potential on Membranes with Fixed Charge
}

\author{
Yuting Sun $^{1}$ and Lianfa Song ${ }^{1}$ \\ ${ }^{1}$ Texas Tech University
}

April 10, 2021

\begin{abstract}
The potential developed on a membrane with fixed charge plays crucial roles in many biological and engineering systems. The classic Teorell-Meyer-Siever (TMS) theory gives an analytical expression of the membrane potential only for limited cases of simple solutions. A numerical method that can be applied to the general cases was developed in this study. With a boundary updating scheme, a numerical solution to the Nernst-Planck-Poisson equations was obtained rigorously without the commonly used simplifications and assumptions in previous studies. The features of the membrane potentials with different fixed charges were investigated with this numerical method under various conditions. The validity of this numerical method was verified by identical values of Donnan potential obtained with well-established analytical methods. The suitability and applicability of analytical TMS model were assessed by comparison to the numerical method.
\end{abstract}

\section{Hosted file}

Manuscript.pdf available at https://authorea.com/users/407054/articles/517477-a-numericalmethod-for-electrical-potential-on-membranes-with-fixed-charge 